\title{
FACTORES AMBIENTALES QUE CONTROLAN LA PRODUCCIÓN DE ESCORRENTÍA Y SEDIMENTO EN EL MATORRAL SEMIÁRIDO DEL SECTOR CENTRAL DE LA DEPRESIÓN DEL EBRO (BAJO VALLE DEL GÁLLEGO, ZARAGOZA). EL PAPEL DE LA PEDREGOSIDAD, LA VEGETACIÓN Y EL SUELO*
}

\author{
María Sebastián ${ }^{1}$, Pedro Sesé ${ }^{1}$, M. T. Echeverría ${ }^{1}$ Teodoro Lasanta², \\ Fernando Pérez-Cabello', Paloma Ibarra ${ }^{1}$ y Juan de la Riva ${ }^{1}$ \\ (1) Dpto. de Geografía y Ordenación del Territorio, Universidad de Zaragoza \\ (2) Instituto Pirenaico de Ecología, CSIC \\ mtecheve@unizar.es
}

\begin{abstract}
Resumen: Se presenta una interpretación del comportamiento hidromorfológico del suelo en el sector central semiárido de la Depresión del Ebro. Se han realizado 25 simulaciones de lluvia en microambientes diferenciados por factores ambientales tales como la pedregosidad, la vegetación o la humedad edáfica. Estas simulaciones se han llevado a cabo en una ladera cubierta por matorral y sometida a pastoreo ocasional. A partir de un análisis estadístico de los resultados obtenidos se ha evaluado el papel estos factores ambientales en el comportamiento hidromorfológico del matorral semiárido.
\end{abstract}

Palabras clave: Escorrentía, producción de sedimento, simulación de lluvia, semiárido, sector central de la Depresión del Ebro.

\begin{abstract}
An interpretation of the soil hydromorphological behaviour in the semi-arid central sector of the Ebro Depression is presented. 25 different simulations of rain in microenvironments with various environmental factors such as vegetation cover, stone density or previous soil moisture have been carried out on a hillside covered by scrubs and subjected to occasional grazing. From a statistical analysis of the results, the influence of the various environmental factors in the hydromorphological behaviour of the semi-arid scrub has been evaluated.
\end{abstract}

Keywords: Runoff, sediment production, rain simulation, semiarid area, central sector of the Ebro Basin.

* Recibido: 9-5-08. Aceptado: 23-1-09. 

Fernando Pérez, Paloma Ibarra y Juan de la Riva

\section{Introducción}

La erosión es uno de los problemas ambientales más importante en el ámbito mediterráneo caracterizado por un ecosistema frágil y sometido a una explotación antrópica intensa en época histórica y a un reciente abandono del suelo, que han contribuido a su degradación (Kosmas et al., 2000). En estos ambientes de marcada irregularidad climática, con lluvias escasas pero intensas, la cubierta vegetal suele jugar un papel fundamental en el control de la erosión, favoreciendo la infiltración del agua en el suelo. Las plantas, además, protegen el suelo del splash e incrementan la cantidad de materia orgánica edáfica, que dificulta la actividad erosiva del agua.

Son muchos los trabajos que han analizado el papel de los factores ambientales en la erosión del suelo en este entorno, como el control topográfico (Cerdà y García Fayos, 1997), la pedregosidad (Yair, 1990; Poesen et al., 1997; González Hidalgo et al., 1999; Cerdà, 2001), las características edáficas (Boix-Fayos et al., 2001), la litología (Imeson et al., 1998; Cerdà, 2002; Calvo et al., 2003; Romero y Belmonte, 2002), el uso del suelo (Bienés y Torcal, 1997; Lasanta et al., 2000) o la cubierta vegetal (Elwell y Stocking, 1976; Thornes, 1990; González Hidalgo et al., 1997, 2000; Sánchez y Puigdefábregas, 1994; Andreu et al., 1998; Bochet et al., 2002, 2006; Ries, 2000; Dunjó et al, 2004...), insistiendo todos ellos en la fragilidad erosiva de los ecosistemas mediterráneos.

En algunos ámbitos del centro de la Depresión del Ebro, el suelo ha permanecido sin cultivar o ha sido abandonado, dedicado en la actualidad al pastoreo ocasional de rebaños de ovino. El matorral cubre con una escasa densidad laderas sobre las que se localizan suelos poco evolucionados, y los efectos de la escorrentía superficial provocan el lavado progresivo de finos, aumentando la pedregosidad y determinando un matorral abierto que realiza una débil, aunque constante, labor como protector del suelo, destacando la eficacia de algunas especies típicamente mediterráneas como Rosmarinus officinalis.

El presente trabajo tiene como objetivo la interpretación del comportamiento hidromorfológico en laderas del semiárido del centro de la Depresión del Ebro a partir de ejercicios de simulación de lluvia. De forma más concreta, se trata de analizar el peso de factores explicativos tales como la vegetación, la pedregosidad y ciertas características edáficas en el proceso de producción de escorrentía superficial y sedimento, teniendo como hipótesis de partida que los mecanismos hidromorfológicos en este tipo de ambientes están controlados por la tasa de infiltración del agua en el suelo.

Este trabajo se incluye en un proyecto de investigación más amplio, que aborda los cambios ambientales a partir de fuegos controlados, en dos ambientes topoclimáticamente contrastados: el sector central de la Depresión del Ebro y el Pirineo Central. 


\section{2. Área de estudio}

El área de estudio está localizado en la margen izquierda del bajo valle del río Gállego, a unos $20 \mathrm{~km}$ al Norte de su desembocadura en el Ebro en la ciudad de Zaragoza (Fig. 1). Según el mapa de estados erosivos de la Cuenca Hidrográfica del Ebro (ICONA, 1987), y siguiendo la aplicación de la U.S.L.E., se trata de un ámbito con pérdidas de suelo que alcanzan valores entre 25 y $50 \mathrm{~T}^{\mathrm{T}} \mathrm{ha}^{-1} \cdot \mathrm{año}^{-1}$, en contraste con la escasa actividad geomorfológica que se detecta en el ámbito de trabajo.
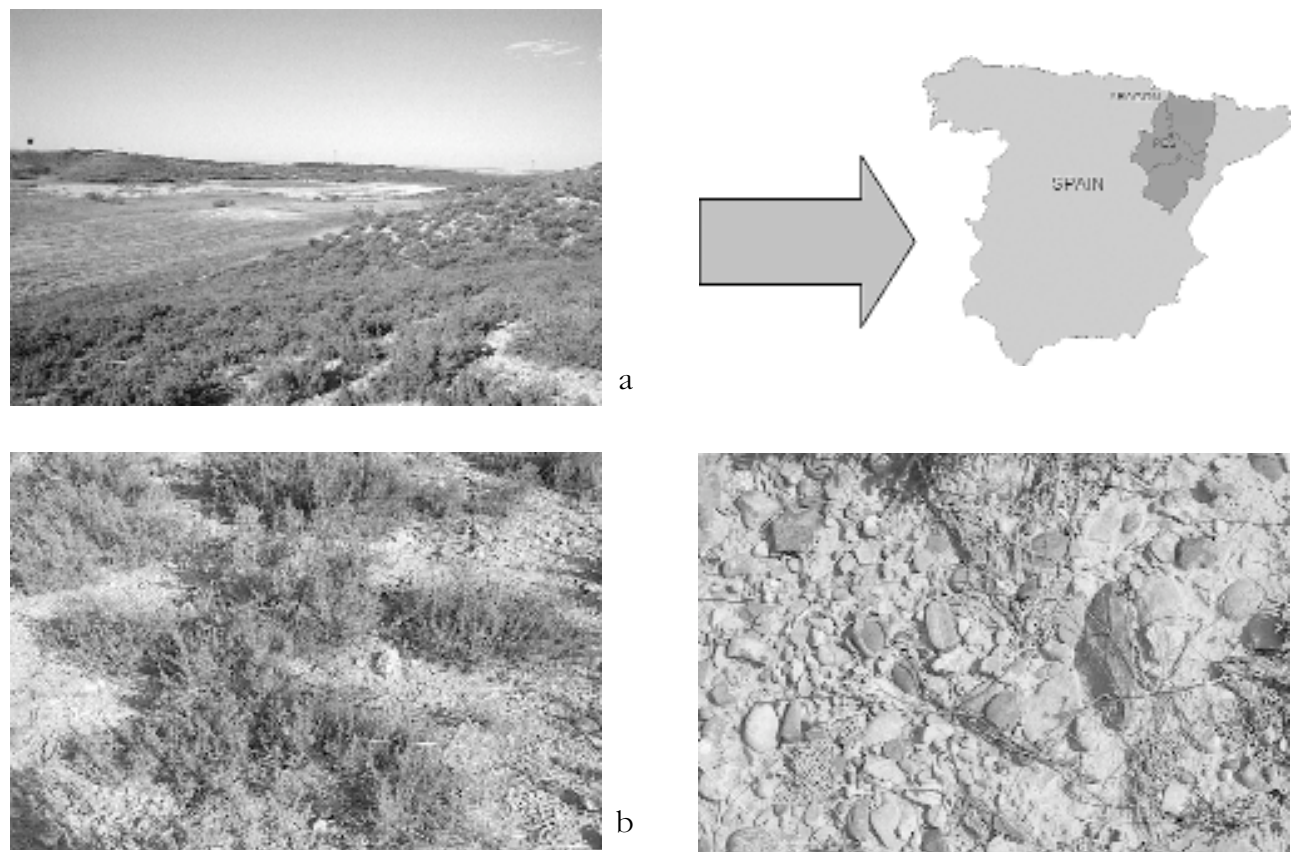

Figura 1. Localización y aspecto general de las laderas y los fondos de valle del área de estudio (Estación Experimental de Peñaflor, PES) (a), distribución de la vegetación (b) y de la pedregosidad con presencia de coprolitos (c).

El área de estudio se asienta sobre una ladera de orientación sur, modelada sobre un sustrato de areniscas y lutitas terciarias con un débil coluvio integrado por cantos de naturaleza fluvial, procedentes de un nivel medio de terraza del río Gállego que fosiliza el sustrato terciario. Sobre la ladera se reconoce un regosol calcárico, cubierto por un matorral de Rosmarinus officinalis y Brachypodium retosum, como especies dominantes (Fot.1). El clima es de tipo mediterráneo seco con precipitaciones anua- 

Fernando Pérez, Paloma Ibarra y Juan de la Riva

les que rondan los $300 \mathrm{~mm}$ y una temperatura media anual de $15^{\circ}$, con fuertes variaciones estacionales. El espacio está sometido a un pastoreo ocasional.

Estas laderas, en conexión con valles de fondo plano ocupados por cultivos de secano, son unidades de paisaje representativas del sector central de la Depresión del Ebro, por lo que el análisis de su comportamiento hidrogeomorfológico contribuye al conocimiento de la transferencia de agua y sedimento entre las laderas y los fondos de valle.

\section{Material y métodos}

Con el objeto de analizar el comportamiento hidrogeomorfológico de la ladera en la que se instala la estación experimental se han llevado a cabo 25 simulaciones de lluvia sobre microparcelas de $0,159 \mathrm{~m}^{2}$, utilizando un simulador semejante al modelo de Calvo et al. (1988).

En cada simulación de lluvia se ha realizado una caracterización de la parcela a partir de valores de pendiente (expresados en \%), cubierta vegetal (\%), pedregosidad superficial (\%), humedad edáfica previa a la simulación (\%) y densidad aparente del suelo (\%). A estos valores se ha añadido la intensidad de precipitación (mm.h-1) utilizada en cada ejercicio (Tabla 1). De forma simultánea, se han analizado diferentes perfiles de suelo a lo largo de la ladera con el objeto de establecer una caracterización textural del mismo. Otros parámetros ambientales, tales como sustrato litológico o exposición topográfica, se han mantenido constantes.

Las simulaciones se han agrupado mediante un análisis cluster a partir de factores ambientales -vegetación, pedregosidad, humedad edáfica previa a la simulación-, intentando definir diferentes microambientes (Fig.2).

Con el objeto de destacar las relaciones entre las variables analizadas, se ha utilizado el coeficiente de correlación de Spearman (Tabla 2), en el que se han contemplado como variables independientes: la pendiente, la vegetación, la pedregosidad, la humedad previa y la intensidad de la precipitación, y como variables-respuesta: la escorrentía, la infiltración, el tiempo de aparición del flujo, la duración de la escorrentía, el coeficiente de escorrentía, la producción de sedimento, la concentración total de sedimento, la cantidad de sólidos en suspensión, la profundidad del frente de humectación y la humedad edáfica posterior a la simulación.

\section{Resultados y discusión}

Los resultados de las simulaciones, relativos a producción de flujo y sedimento, se han expresado en la Tabla 3 y en gráficas que recogen los valores acumulados de escorrentía superficial, concentración total de sedimento y sólidos en suspensión (Gráficas 1). La producción, tanto de flujo como de sedimento, es en general escasa 
Tabla 1. Características ambientales de las microparcelas de simulación de lluvia (grupo 1, gris claro; grupo 2, gris oscuro; grupo 3, blanco).

\begin{tabular}{|c|c|c|c|c|c|c|}
\hline \multirow{26}{*}{ 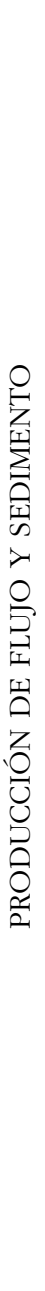 } & $\begin{array}{l}\text { Simu- } \\
\text { lación }\end{array}$ & $\begin{array}{c}\text { Pendiente } \\
\text { (\%) }\end{array}$ & $\begin{array}{c}\text { Cubierta } \\
\text { vegetal } \\
\text { (\%) }\end{array}$ & $\begin{array}{l}\text { Pedrego- } \\
\text { sidad } \\
\text { (\%) }\end{array}$ & $\begin{array}{l}\text { Humedad } \\
\text { edáfica } \\
\text { previa (\%) }\end{array}$ & $\begin{array}{c}\text { Intensidad } \\
\text { precipitación } \\
\text { ( } \mathrm{mm})\end{array}$ \\
\hline & 1 & 14 & 14 & 62 & 8,7 & 37,4 \\
\hline & 2 & 14 & 2 & 72 & 9,9 & 37,4 \\
\hline & 3 & 13 & 5 & 90 & 9,1 & 31,8 \\
\hline & 4 & 18 & 95 & 3 & 23,2 & 68,6 \\
\hline & 5 & 18 & 90 & 2 & 7,2 & 24,1 \\
\hline & 6 & 24 & 95 & 15 & 5,5 & 44,4 \\
\hline & 7 & 13,5 & 40 & 85 & 8,7 & 41,3 \\
\hline & 8 & 18 & 55 & 95 & 6,8 & 40,5 \\
\hline & 9 & 20 & 5 & 90 & 17,2 & 72,3 \\
\hline & 10 & 16 & 70 & 20 & 12,4 & 63,1 \\
\hline & 11 & 15 & 2 & 95 & 12,7 & 42,9 \\
\hline & 12 & 20 & 55 & 40 & 11,6 & 39,0 \\
\hline & 13 & 17 & 3 & 95 & 12,6 & 45,2 \\
\hline & 14 & 20 & 70 & 85 & 12,7 & 38,2 \\
\hline & 15 & 21 & 25 & 85 & 11,3 & 53,8 \\
\hline & 16 & 10 & 100 & 15 & 17,3 & 37,8 \\
\hline & 17 & 10 & 55 & 75 & 19,5 & 52,0 \\
\hline & 18 & 14 & 10 & 90 & 12,8 & 64,3 \\
\hline & 19 & 16 & 47 & 90 & 19,1 & 44,0 \\
\hline & 20 & 15 & 50 & 10 & 34,4 & 36,6 \\
\hline & 21 & 11 & 95 & 5 & 12,3 & 36,0 \\
\hline & 22 & 11 & 15 & 95 & 13,5 & 48,3 \\
\hline & 23 & 12 & 55 & 95 & 16,6 & 54,1 \\
\hline & 24 & 18 & 70 & 80 & 11,1 & 51,4 \\
\hline & 25 & 13 & 10 & 90 & 12,9 & 52,8 \\
\hline
\end{tabular}

arrojando valores medios de 21,09\% para el coeficiente de escorrentía y de 1,36 gr/1 para la concentración de sedimento. Este comportamiento hidrogeomorfológico es moderado en comparación con otros ámbitos del sector central de la Depresión del Ebro, como el bajo valle del Huerva, donde se alcanzan coeficientes de escorrentía del 76\% (Ries, 2003) o los relieves en yesos del sector central de la Depresión del Ebro con coeficientes de escorrentía entre 30 y $64 \%$ y concentraciones de sedimento 

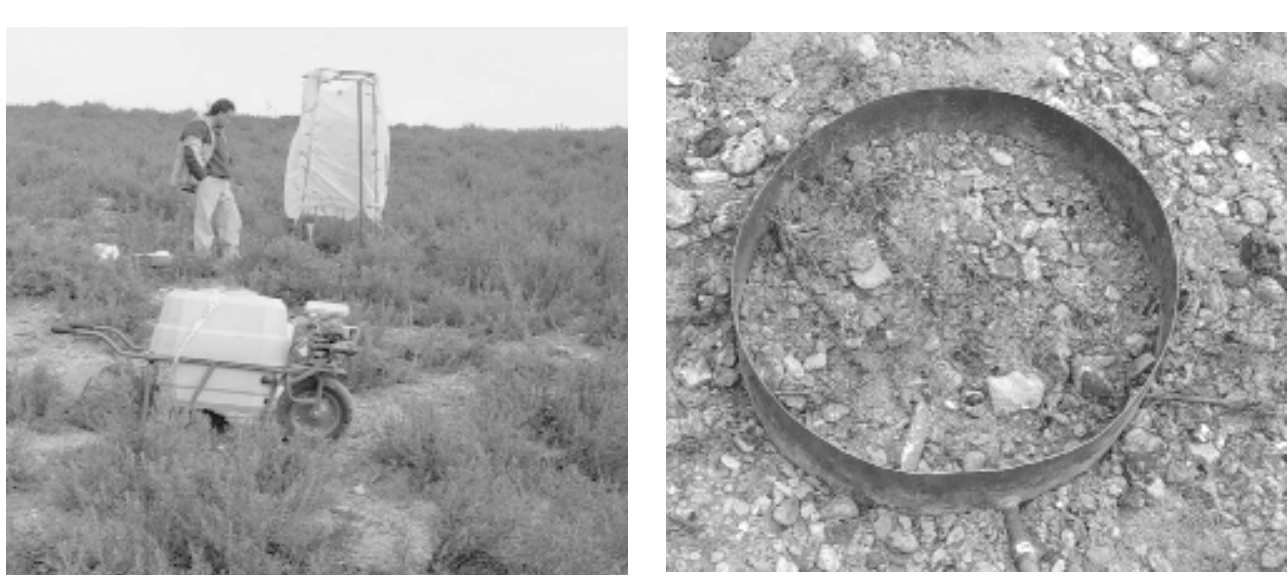

Figura 2. Equipo y parcela de simulación.

de 4,35 gr/l (Desir, 2001). Los bajos valores de escorrentía en el área de estudio pueden explicarse por una textura edáfica, mayoritariamente arenosa (20-50\% de arenas), así como por la presencia estacional pero abundante de coprolitos de lombrices que aceleran los proceso de infiltración en el suelo, impidiendo la escorrentía superficial en algunas simulaciones.

A partir de parámetros de caracterización de las parcelas tales como pedregosidad, cubierta vegetal, humedad edáfica e intensidad de la lluvia -se han desechado la pendiente y la densidad aparente del suelo por su escasa variabilidad- las simulaciones han sido agrupadas, definiendo mediante el análisis de tipo clúster, tres ambientes homogéneos (Fig.2), cuyos estadísticos de grupo se recogen en la Tabla 4.

- Primer grupo (simulaciones $\mathrm{n}^{\circ}$ 1, 2, 3, 7, 11, 13, 17, 18, 19, 22, 23, 25) caracterizado por los siguientes valores medios: una pedregosidad elevada (86\%), elevadas intensidades de precipitación $\left(46 \mathrm{~mm} \cdot \mathrm{h}^{-1}\right)$, escasa cubierta vegetal (21\%) y una humedad edáfica previa semejante al resto de los grupos (13,04\%). Este primer grupo incluye prácticamente la mitad de las simulaciones y, bajo estas condiciones, los valores que caracterizan la respuesta hidrogeomorfológica son los siguientes: tiempo de aparición de la escorrentía, $4,77 \mathrm{mn}$; frente de humectación, 13,08 cm; coeficiente de escorrentía, 26,52\%; concentración de sedimento, 2,24 gr/1; producción total de sólidos, 27,4 gr.

- Segundo grupo (simulaciones no 4, 5, 6, 8, 9, 10, 12, 14, 15, 24) caracterizado por una pedregosidad media (51\%),más altas intensidades de precipitación (49,5 $\mathrm{mm} \cdot \mathrm{h}^{-1}$ ), densa cubierta vegetal (63\%) y una humedad edáfica previa semejante al resto de los grupos (12,01\%). Este segundo grupo incluye un total de 9 simulaciones en las que los valores que caracterizan la respuesta hidrogeomorfoló- 
Tabla 2. Rho de Spearman. (*) La correlación es significativa al nivel 0,05; (**). La correlación es significativa al nivel 0,01.

\begin{tabular}{|c|c|c|c|c|c|}
\hline \multicolumn{6}{|c|}{ Coeficiente Rho de Spearman } \\
\hline Variables & Pendiente & Vegetación & Pedregosidad & $\begin{array}{c}\text { Humedad } \\
\text { previa del } \\
\text { suelo }\end{array}$ & Intensidad \\
\hline Humedad previa del suelo & $\begin{array}{l}, 037 \\
, 867\end{array}$ & $\begin{array}{r}-, 103 \\
, 639\end{array}$ & $\begin{array}{l}, 022 \\
, 922\end{array}$ & $\begin{array}{r}, 487^{*} \\
, 018\end{array}$ & $\begin{array}{l}, 188 \\
, 392\end{array}$ \\
\hline Densidad de suelo & $\begin{array}{l}, 398 \\
, 060\end{array}$ & $\begin{array}{r}-, 246 \\
, 257\end{array}$ & $\begin{array}{r}-, 034 \\
, 877 \\
\end{array}$ & $\begin{array}{r}-, 532^{*} \\
, 099\end{array}$ & $\begin{array}{r}-, 079 \\
, 719\end{array}$ \\
\hline Escorrentía superficial & $\begin{array}{r}-, 006 \\
, 978\end{array}$ & $\begin{array}{r}-, 575^{* *} \\
, 004\end{array}$ & $\begin{array}{l}, 104 \\
, 635\end{array}$ & $\begin{array}{r}-, 053 \\
, 809\end{array}$ & $\begin{array}{r}-, 054 \\
, 805\end{array}$ \\
\hline Infiltración & $\begin{array}{l}, 072 \\
, 744\end{array}$ & $\begin{array}{l}343 \\
, 109\end{array}$ & $\begin{array}{l}, 015 \\
, 944\end{array}$ & $\begin{array}{l}, 361 \\
, 091\end{array}$ & $\begin{array}{r}, 847^{*} \\
, 000\end{array}$ \\
\hline Tiempo aparición del flujo & $\begin{array}{r}-, 100 \\
, 651\end{array}$ & $\begin{array}{l}294 \\
, 173\end{array}$ & $\begin{array}{l}, 113 \\
, 608\end{array}$ & $\begin{array}{r}-, 002 \\
, 991\end{array}$ & $\begin{array}{r}-, 170 \\
, 438\end{array}$ \\
\hline Duración de la escorrentía & $\begin{array}{l}, 100 \\
, 651\end{array}$ & $\begin{array}{l}, 294 \\
, 173\end{array}$ & $\begin{array}{r}-, 113 \\
, 608\end{array}$ & $\begin{array}{r}-, 002 \\
991\end{array}$ & $\begin{array}{r}-, 170 \\
, 438\end{array}$ \\
\hline Coeficiente de escorrentía & $\begin{array}{r}-, 026 \\
, 907\end{array}$ & $\begin{array}{r}-, 605^{* *} \\
, 002\end{array}$ & $\begin{array}{l}, 093 \\
, 672\end{array}$ & $\begin{array}{r}-, 145 \\
, 508\end{array}$ & $\begin{array}{r}-, 205 \\
, 349\end{array}$ \\
\hline Producción de sedimento & $\begin{array}{r}-, 014 \\
, 951\end{array}$ & $\begin{array}{r}-, 516^{*} \\
, 012\end{array}$ & $\begin{array}{r}-, 031 \\
, 887\end{array}$ & $\begin{array}{r}-, 125 \\
, 571\end{array}$ & $\begin{array}{r}-, 131 \\
, 551\end{array}$ \\
\hline Frente de humectación & $\begin{array}{l}, 190 \\
, 385\end{array}$ & $\begin{array}{r}-, 131 \\
, 551\end{array}$ & $\begin{array}{l}, 155 \\
, 480\end{array}$ & $\begin{array}{l}, 128 \\
, 560\end{array}$ & $\begin{array}{l}, 173 \\
, 429\end{array}$ \\
\hline $\begin{array}{l}\text { Concentración del } \\
\text { sedimento }\end{array}$ & $\begin{array}{l}, 133 \\
, 545\end{array}$ & $\begin{array}{r}-, 214 \\
, 327\end{array}$ & $\begin{array}{r}-, 238 \\
, 274\end{array}$ & $\begin{array}{r}-, 376 \\
, 077\end{array}$ & $\begin{array}{r}-, 216 \\
, 322\end{array}$ \\
\hline Sólidos en suspensión & $\begin{array}{l}, 067 \\
, 760\end{array}$ & $\begin{array}{r}-, 496^{*} \\
, 016\end{array}$ & $\begin{array}{l}051 \\
, 816\end{array}$ & $\begin{array}{r}-, 318 \\
, 139\end{array}$ & $\begin{array}{r}-, 276 \\
, 203\end{array}$ \\
\hline
\end{tabular}

gica son los siguientes: tiempo de aparición de la escorrentía, 6,1 mn; frente de humectación, 15,83 cm; coeficiente de escorrentía, 15,06\%; concentración de sedimento, 1,75gr/1; producción total de sólidos, 10,66 gr.

- Tercer grupo (simulaciones $n^{\circ}$ 16, 20, 21) caracterizado por una pedregosidad muy baja (10\%), menores intensidades de precipitación $\left(36,8 \mathrm{~mm} \cdot \mathrm{h}^{-1}\right)$, muy densa cubierta vegetal $(81,66 \%)$ y una humedad edáfica previa semejante al resto de las parcelas. La falta de alguno de los datos en una serie tan corta, 3 simulaciones, no permite extraer conclusiones representativas del grupo.

Parece deducirse que la presencia de una elevada pedregosidad y la menor cubierta vegetal del grupo 1 (Gráficas 1a), adelantan el tiempo de respuesta de la escorrentía, aumentan el flujo superficial, así como la concentración y producción total de sedimento, reduciendo el frente de humectación; por el contrario, la menor 

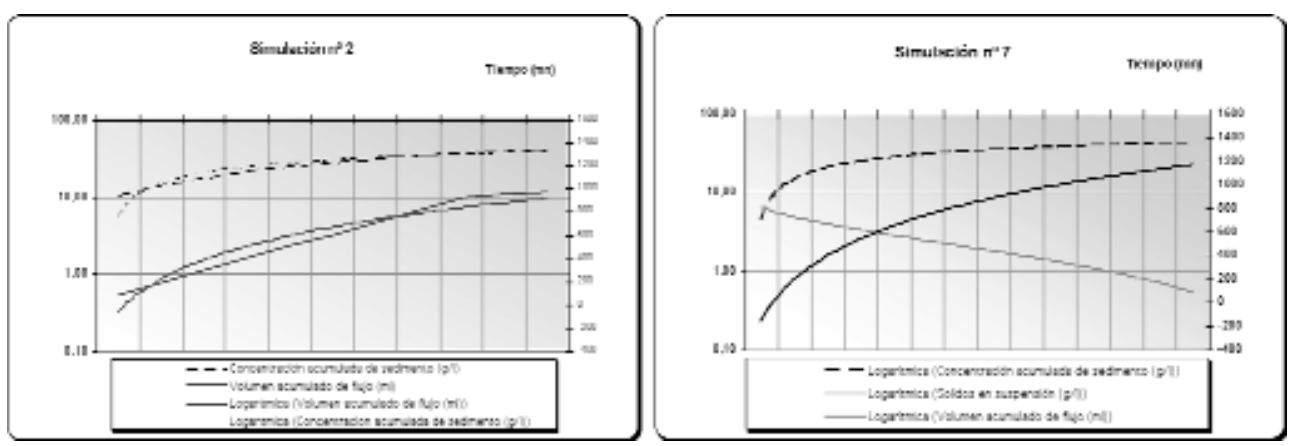

a) Grupo 1
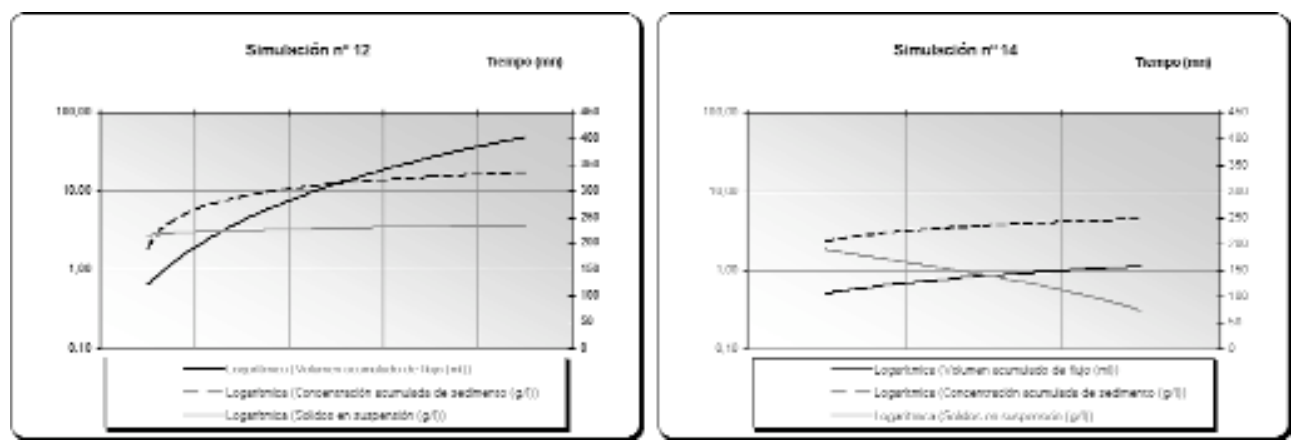

b) Grupo 2

Gráfica 1. Gráficas de producción de flujo y sedimento correspondientes a los grupos definidos a partir del Cluster: a) Grupo 1; b) Grupo 2.
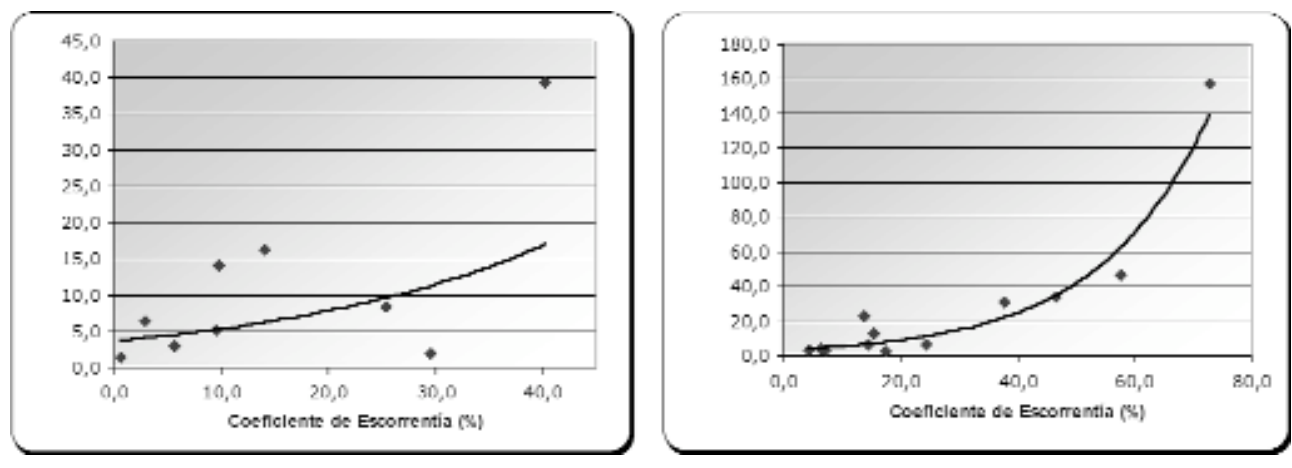

Gráfica 2. Relación entre Coeficiente de Escorrentía y Producción total de Sedimento en el Grupo 1 (izquierda) y Grupo 2 (derecha). 
Tabla 3. Valores de producción de flujo y sedimento (concentración y producción total) y coeficientes de escorrentía e infiltración en las simulaciones de lluvia.

\begin{tabular}{|c|c|c|c|c|c|c|c|}
\hline & 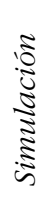 & 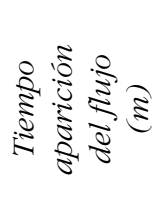 & 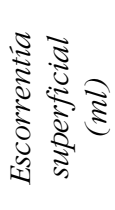 & 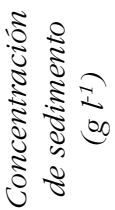 & 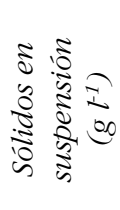 & 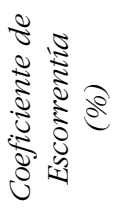 & 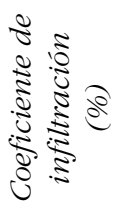 \\
\hline \multirow{25}{*}{ 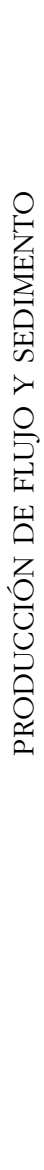 } & 1 & 1,5 & 1887 & 7,9 & 157,3 & 72,8 & 27,2 \\
\hline & 2 & 1,5 & 980 & 2,8 & 30,8 & 37,8 & 62,1 \\
\hline & 3 & 8,8 & 538 & 1,0 & 6,2 & 24,3 & 75,6 \\
\hline & 4 & 2,6 & 1406 & 0,1 & 1,9 & 29,5 & 70,4 \\
\hline & 5 & 11,9 & 0 & 0 & 0 & 0 & 0 \\
\hline & 6 & 8,7 & 88 & 3,2 & 6,4 & 2,8 & 97,1 \\
\hline & 7 & 4,7 & 1330 & 2,4 & 33,6 & 46,4 & 53,5 \\
\hline & 8 & 5,4 & 714 & 1,0 & 8,4 & 25,4 & 74,5 \\
\hline & 9 & 2,3 & 2017 & 2,1 & 39,2 & 40,2 & 59,7 \\
\hline & 10 & 6,0 & 30 & 1,4 & 1,4 & 0,6 & 99,3 \\
\hline & 11 & 2,7 & 1710 & 2,9 & 46,6 & 57,5 & 42,4 \\
\hline & 12 & 1,5 & 381 & 3,2 & 16,2 & 14,1 & 85,8 \\
\hline & 13 & 10,3 & 453 & 1,2 & 6,2 & 14,4 & 85,5 \\
\hline & 14 & 14,5 & 151 & 0,9 & 2,9 & 5,7 & 93,2 \\
\hline & 15 & 5,2 & 357 & 1,3 & 5,2 & 9,5 & 90,4 \\
\hline & 16 & 19,0 & 34 & 0,1 & 0,2 & 1,3 & 98,7 \\
\hline & 17 & 3,7 & 503 & 3,7 & 22,6 & 13,9 & 86,1 \\
\hline & 18 & 4,0 & 688 & 1,6 & 12,8 & 15,4 & 84,5 \\
\hline & 19 & 2,7 & 217 & 0,8 & 3,2 & 7,1 & 92,8 \\
\hline & 20 & 6,1 & 702 & 2,7 & 18,9 & 27,6 & 72,3 \\
\hline & 21 & 0 & 0 & 0 & 0 & 0 & 0 \\
\hline & 22 & 7,0 & 213 & 1,1 & 3,5 & 6,3 & 93,6 \\
\hline & 23 & 5,2 & 167 & 0,9 & 2,9 & 4,4 & 95,5 \\
\hline & 24 & 3,8 & 347 & 2,2 & 14,1 & 9,7 & 90,2 \\
\hline & 25 & 5,2 & 642 & 0,7 & 2,7 & 17,5 & 82,4 \\
\hline
\end{tabular}

pedregosidad y la mayor superficie de cubierta vegetal (Gráficas 1b) retrasan el tiempo de respuesta del flujo, reducen la escorrentía superficial, la concentración y producción total de sedimento, y alcanza mayor profundidad el frente de humectación. Además, las condiciones ambientales del grupo 1, mayor pedregosidad y menor 
cubierta vegetal, sometidas a valores más intensos de precipitación, dan lugar a una relación positiva entre el coeficiente de escorrentía y la producción total de sedimento (Gráfica 2).

A partir de los resultados derivados del coeficiente de correlación de Spearman (Tabla 3) destacan, por un lado, la escasa capacidad explicativa que tienen algunos factores como la pendiente -cuya variabilidad, por otro lado, es escasa- la pedregosidad o la humedad edáfica previa y, por el contrario, las fuertes correlaciones entre la cubierta vegetal y la génesis de escorrentía superficial y, en menor medida, la producción de sedimento.

Tabla 4. Estadísticos de grupo.

\begin{tabular}{|c|l|r|r|r|}
\hline \multicolumn{5}{|c|}{ Estadísticos de grupo } \\
\hline \multirow{6}{*}{ Grupos } & Método de War & Media & $\begin{array}{c}\text { Desviación } \\
\text { Típica }\end{array}$ & $\begin{array}{c}\text { Número } \\
\text { de valores } \\
\text { ponderados }\end{array}$ \\
\hline \multirow{6}{*}{1} & Pendiente & 13,5 & 1,9 & \\
& Cubierta vegetal & 21,5 & 21,2 & 12 \\
& Pedregosidad & 86,1 & 10,7 & \\
& Humedad previa & 13,0 & 3,7 & \\
& Intensidad & 46,0 & 8,9 & \\
\hline \multirow{5}{*}{2} & Pendiente & 19,3 & 2,2 & \\
& Cubierta vegetal & 63,0 & 29,5 & \\
& Pedregosidad & 51,5 & 39,0 & \\
& Humedad previa & 11,9 & 5,2 & \\
& Intensidad & 49,5 & 15,1 & \\
\hline \multirow{6}{*}{3} & Pendiente & 12,0 & 2,6 & \\
& Cubierta vegetal & 81,6 & 27,5 & \\
& Pedregosidad & 10,0 & 5,0 & \\
& Humedad previa & 21,3 & 11,6 & \\
& Intensidad & 36,8 & 0,9 & \\
\hline \multirow{6}{*}{ TOTAL } & Pendiente & 15,6 & 3,6 & \\
& Cubierta vegetal & 45,3 & 34,3 & \\
& Pedregosidad & 63,1 & 36,0 & \\
& Humedad previa & 13,6 & 6,0 & \\
& Intensidad & 46,3 & 11,7 & \\
\hline
\end{tabular}

\section{Conclusiones}

Las formaciones de matorral asentadas sobre suelos fácilmente erosionables son frecuentes en ambientes mediterráneos y los eventos de lluvia con intensidades en 
torno a los $40 \mathrm{~mm} \cdot \mathrm{h}^{-1}$, tienen una importante capacidad erosiva en estos ámbitos. En el área de estudio la producción de sedimento y, de forma más marcada, la de flujo está controlada, además de por la textura arenosa de los suelos y la presencia de abundantes coprolitos, por el porcentaje de cubierta vegetal, responsable de la reducción de la escorrentía superficial, al facilitar los procesos de infiltración hídrica en el suelo. Si bien es reconocida la eficacia de la actividad protectora del Rosmarinus officinalis (Bochet et al., 2006), especie vegetal dominante en el área de estudio, su patrón de ocupación en forma de individuos aislados, no es capaz de frenar los procesos relacionados con la erosión hídrica.

Estas laderas cubiertas por matorral discontinuo son una unidad paisajística muy característica del sector central de la Depresión del Ebro y se constituyen como fuentes productivas de sedimento y escorrentía. Las laderas tienen como nivel de base local valles de fondo plano, ocupados por cultivos de secano. Si, como en el área de estudio, los suelos presentan una moderada capacidad de infiltración, la vegetación se convierte en un factor que regula el comportamiento hidrogeomorfológico, con una escasa movilización de flujo y sedimento hacia los fondos de valle.

No obstante, la variabilidad de las relaciones establecidas entre los condicionantes ambientales demanda una continuidad en este tipo de trabajos, combinando diferentes escalas experimentales, la microescala representada por simulación de lluvia sobre parcelas en torno a $1 \mathrm{~m} 2$, la mesoescala, utilizando parcelas de erosión o la macroescala, disponiendo de cuencas de drenaje monitorizadas.

\section{Agradecimientos}

El presente trabajo se ha podido llevar a cabo mediante la ayuda económica de los proyectos REN2002-00133/GLO del Ministerio de Ciencia y Tecnología, PIP098/2005 del Gobierno de Aragón y CGL2005-04863/CLI y CGL2007-66644-C04 del Ministerio de Ciencia y Tecnología.

\section{Referencias bibliográficas}

Andreu, V., Rubio, J.L. y Cerni, R. (1998) Effects of Mediteranean shrub on water erosion control. Environmental Monitoring and Assesment, 37: 5-15.

Bienés, R. y Torcal, L. (1997) Influencia del manejo del suelo sobre la erosión en depósitos de terraza (El Encín y Marchamalo). Cuaternario y Geomor-fología, 11: 113-124.
Bochet, E., Rubio, J.L. y Poesen, J. (1998) Relative efficiency of three representative matorral species in reducing water erosion at the microscale in a semi-arid climate (Valencia, Spain). Geomorphology, 23: 139150.

Bochet, E., Poesen, J. y Rubio, J.L. (2002) Influence of plant morphology on splash erosion in a Mediterranean matorral. 

Fernando Pérez, Paloma Ibarra y Juan de la Riva

Zeitschrift für Geomorphologie Neu Folge, 46: 223-243.

Bochet, E., Poesen, J. y Rubio, J.L. (2006) Runoff and soil loss under individual plants of a semi-arid Mediterranean shrubland: influence of plant morphology and rainfall intensity. Earth Surface Processes and Landforms, 31: 536-549.

Boix-Fayos, C., Calvo Cases, A., Imeson, A. C. y Soriano Sdoto, M.D. (2001) Influence of soil properties on the agregation of some Mediterranean soils and the use of aggregate size and stability as land degradation indicators. Catena, 44: 47-67.

Calvo, A., Gisbert, J.M., Palau, E. y Romero, M. (1988) Un simulador de lluvia de fácil construcción. In M. Sala y F. Gallart (eds.) Métodos y técnicas para la medición en el campo de procesos geomorfológicos. Monografía 1, 6-15. S.E.G.

Calvo, A., Boix, C. e Imeson, C. (2003) Runoff generation, sediment movement and soil water behaviour on calcareous (limestone) slopes of some Mediterranean environments in southeast Spain. Geomorphology, 50: 269-291

Cerdà, A. y García Fayos, P. (1997) The influence of slope angle on sediment, water and seed losses on badlands landscapes. Geomorphology, 18: 77-90.

Cerdà, A. (2001) Effects of rock fragment cover on soil infiltration, interril runoff and erosion. European Journal of Soil Science, 52: 59-68.

Cerdà, A. (2002) The effect of season and parent material on water erosion on highly eroded soils in Eastern Spain. Journal of Arid Environmets, 52: 319-337.

Desir, G. (2001) Erosión hidrica de terrenos yesíferos en el sector central de la Depresión del Ebro. Zaragoza, Consejo de Protección de la Naturaleza de Aragón, $326 \mathrm{p}$.

Dunjó, G., Pardini, G. y Gispert, M. (2004) The role of land use-land cover on runoff generation and sediment yield at a microplot scale, in a small Mediterranean catchment. Journal of Arid Environments, 57: 94-116.

Elwell, H.A. y Stocking, M.A. (1976) Vegetal cover to estimate soil erosion hazard in Rhodesia. Geoderma, 15: 61-70.

González Hidalgo, J.C., Raventos, J. y Echeverría, M.T. (1997) Comparation of sediment ratio curves for plants with different architectures. Catena, 29: 333-340.

González Hidalgo, J.C., Echeverría, M.T. y Vallejo, R.V. (1999) Selective transport of sediment related to rainfall kinetic energy, plant cover-stoniness and clearing. Zeitschrift für Geomorphologie Neu Folge, 43: 255-266.

ICONA (1987) Mapas de Estados Erosivos. Cuenca Hidrográfica del Ebro. ICONA. 87 p. + anexo cartográfico.

Imeson, A.C., Lavee, H., Calvo, A. y Cerdà, A. (1998) The erosional response of calcareous soils along a climatological gradient in Southeast Spain. Geomorphology, 24: 3-16.

Kosmas, C., Danalatos, N.G. y Gerontidis, S. (2000) The effect of land parameters on vegetation performance and degree of erosion under Mediterranean conditions. Catena, 40: 3-17.

Lasanta, T., García Ruiz, J.M., Pérez Rontomé, C. y Sancho, C. (2000) Runoff and sediment yield in a semiarid environment. The effect of land management after farmland abandonment. Catena, 38: 265-278.

Poesen, J., Van Wesemael, B, Govers, G., Martínez-Fernández, J., Desmet, P., Vandaele, K., Quine, T. y Degraer, G. (1997) Patterns of rock fragment cover generated by tillage erosion. Geomorphology, 18: 183-197.

Ries, J.B. (2000) Experimental investigations on water and wind erosion on abandone fields and arable land in the central Ebro basin, Aragon, Spain. Zeitschrift für Geomorphologie, Supple. Band., 121: 91108.

Ries, J.B. (2003) Land use change and land degradation in Spain. A short introduction. In Marzolff, I., Ries, J.B., de la Riva, J. y 
Seeger, M (coords.) El cambio en el uso del suelo y la degradación del territorio en España, 11-29, Johann Wolfgang GoetheUniversität Frankfurt am Main y Universidad de Zaragoza

Romero, A. y Belmonte, F. (2002) Erosión de suelo en ambiente semiárido extremo bajo diferentes tipos de litología y suelos. En A. Pérez González, J. Vegas y M. Machado (eds.) Aportaciones a la Geomorfología de España en el Inicio del Tercer Milenio, 315-322. IGME.
Sánchez, G. y Puigdefábregas, J. (1994) Interactions of plant growth and sediment movement on slopes in a semiarid environment. Geomorphology, 9: 243-260.

Thornes, J.B. (1990) The interaction of erosional and vegetational dynamics in land degradation: spatial outcomes. In Thornes, J.B. (ed.): Vegetation and erosion, 45-53. Chichester, Wiley.

Yair, A. (1990) The role of topography and surface cover upon soil formation along hillslopes in arid climates. Geomorphology, 3: 287-299. 


\section{Figuras y Tablas}
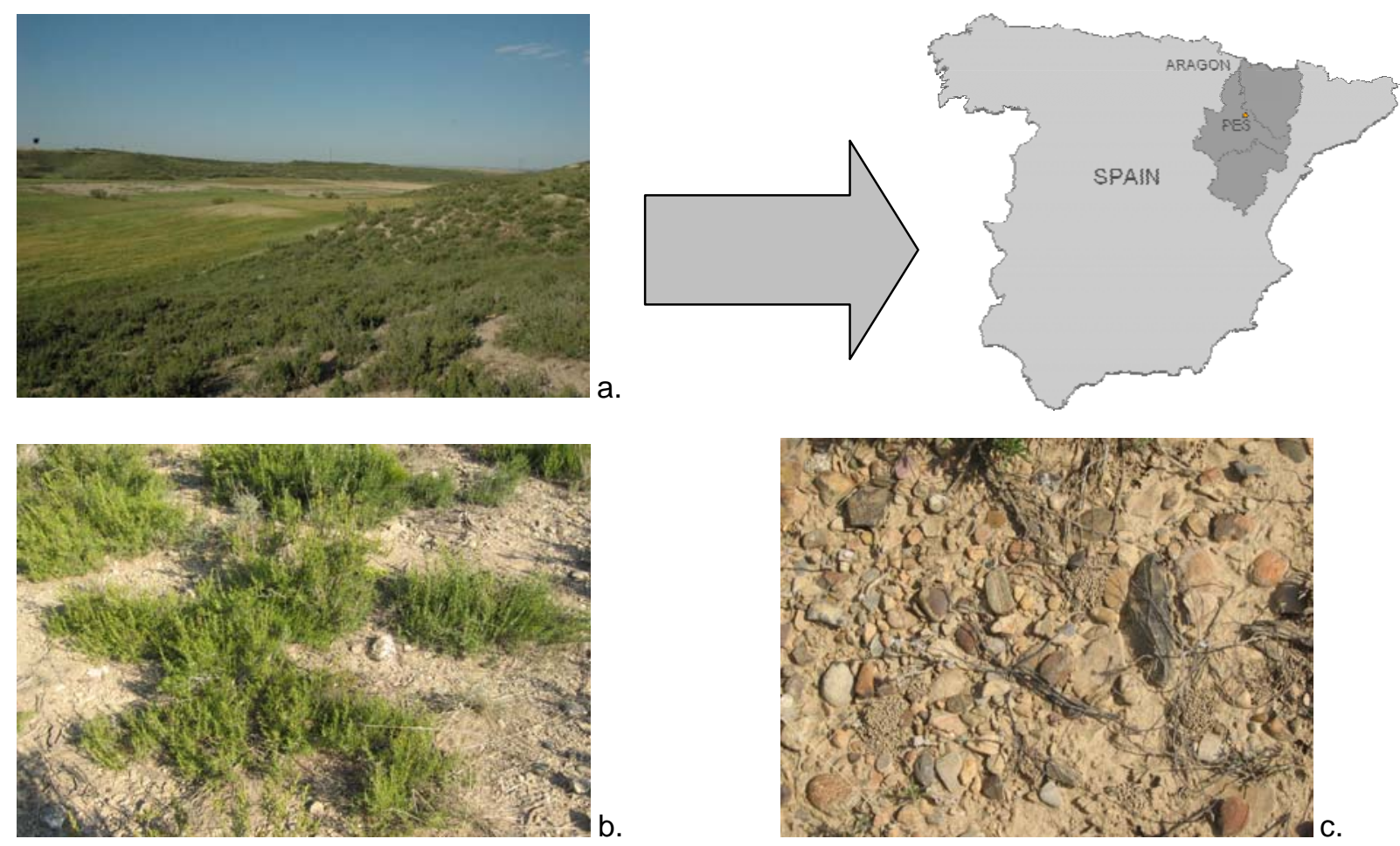

Figura 1.- Localización y aspecto general de las laderas y los fondos de valle del área de estudio (Estación Experimental de Peñaflor, PES) (a), distribución de la vegetación (b) y de la pedregosidad con presencia de coprolitos (c).
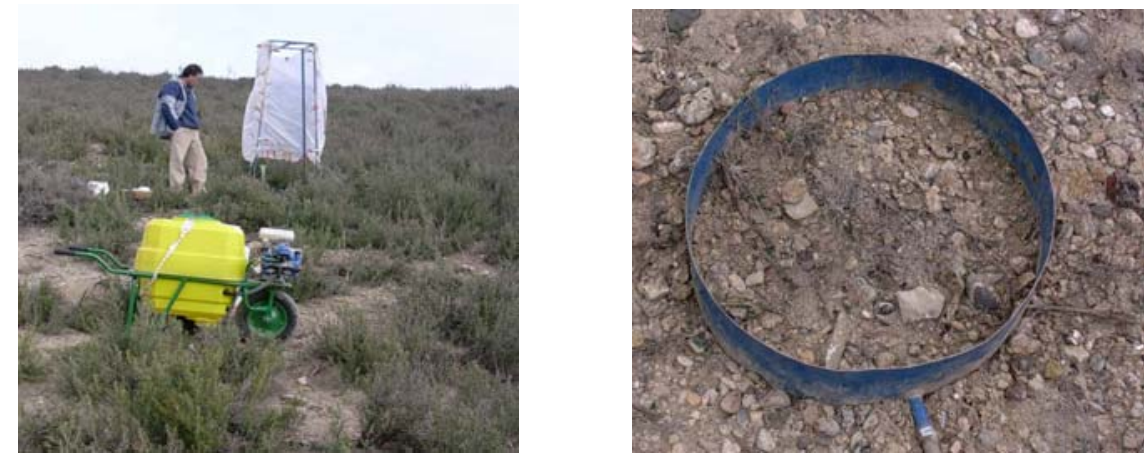

Figura 2.- Equipo y parcela de simulación. 